\title{
DETERMINACIÓN DE LA DISTRIBUCIÓN DE CONTAMINANTES EMERGENTES EN AGUA INTERSTICIAL EN SEDIMENTOS DE HUMEDAL MEDIANTE LA OPTIMIZACIÓN Y VALIDACIÓN DE UN MÉTODO ANALÍTICO
}

\author{
(Determination of the distribution of emerging pollutants in interstitial water from wetland \\ sediments through the optimization and validation of an analytical method) \\ Manuel HERNÁNDEZ-QUIROZ ${ }^{1}$, Diego RUIZ-MEZA ${ }^{1}$, \\ Francisco ROJO-CALLEJAS ${ }^{2}$ y Claudia PONCE DE LEÓN-HILL ${ }^{1 *}$ \\ ${ }^{1}$ Área de Biología, Facultad de Ciencias, Universidad Nacional Autónoma de México, Circuito Exterior s/n, \\ ${ }^{2}$ Posgrado de Ciencias del Mar y Limnología, Universidad Nacional Autónoma de México, Circuito Exterior
} Ciudad Universitaria, 04510 Ciudad de México, México s/n, Ciudad Universitaria, 04510 Ciudad de México, México

*Autor para correspondencia: caplh@ciencias.unam.mx

(Recibido marzo 2018; aceptado agosto 2018)

Palabras clave: productos farmacéuticos activos, agua de poro, Xochimilco, cromatografía de gases acoplada a espectrometría de masas

\section{RESUMEN}

El presente trabajo muestra la metodología (estudio de un método) para el análisis de cuatro contaminantes emergentes. Cada contaminante emergente estudiado, representa a una familia de interés de los contaminantes emergentes (un compuesto estrogénico, uno antibacteriano, un fármaco ácido y una fragancia). El método se optimizó para ser utilizado en una matriz ambiental compleja, como el agua intersticial de sedimentos de un humedal con alto contenido de materia orgánica. La extracción de los compuestos se llevó a cabo mediante la técnica de extracción en fase sólida y se cuantificó mediante cromatografía de gases con espectrometría de masas en modo de monitorización de ion único. El método se ratificó utilizando el "Proceso de validación del método de laboratorio único" mediante la determinación de los parámetros de validación del método. El método optimizado se aplicó al análisis del agua intersticial de los sedimentos de los canales de la zona agrícola periurbana de Xochimilco, México. Este estudio preliminar mostró que el punto de muestreo cercano al área de descarga de aguas residuales de la planta de tratamiento de aguas residuales de tratamiento secundario tenía la mayor concentración de contaminantes emergentes. Además, se observó una relación inversamente proporcional entre las concentraciones de ibuprofeno y almizcle cetona y la concentración de materia orgánica en los sedimentos. La optimización del método y la ratificación con muestras de agua intersticial de sedimentos del humedal, mostraron su valor para la evaluación de la presencia y distribución de contaminantes emergentes.

Kew words: active pharmaceutical compounds, pore water, Xochimilco, gas chromatography coupled to mass spectrometry 


\begin{abstract}
The present work shows the methodology (study of a method) for the analysis of four emerging pollutants. Each studied pollutant represents a family from emerging pollutants (an estrogenic compound, an antibacterial, a pharmaceutical and a fragrance). The method was optimized to be used in a complex environmental matrix such as sediment interstitial water of a wetland with high organic matter content. The extraction of the compounds was carried out by solid phase extraction technique and quantified by gas chromatography with mass spectrometry in single ion monitoring mode. The method was validated using the "Single Laboratory Method Validation Process" through the determination of the performance parameters of the method. The optimized method was applied to the analysis of sediment interstitial water from the canals of the periurban agricultural zone of Xochimilco, Mexico. This preliminary study showed that the sampling point close to the wastewater discharge area from the secondary treatment wastewater plant had the highest concentration of emerging pollutants. Also, an inversely proportional relationship was observed between the concentrations of ibuprofen and musk with the concentration of organic matter in the sediments. The optimization of the method and the ratification with sediment interstitial water samples from the wetland, showed the worthiness of the method for the evaluation of the presence and distribution of emerging pollutants.
\end{abstract}

\section{INTRODUCCIÓN}

Los contaminantes emergentes han llamado la atención desde mediados del siglo XX al representar un riesgo potencial para el ambiente y para la salud. Como contaminante emergente (CE) se entiende todo aquel previamente desconocido o no reconocido como tal, cuya presencia en el ambiente no es necesariamente nueva pero que su persistente presencia y/o concentración, causa preocupación por las posibles consecuencias ambientales y a la salud humana. También se han definido como contaminantes no regulados, que pueden ser candidatos para la regulación futura dependiendo de la investigación sobre sus potenciales efectos de salud y datos de monitoreo con respecto a su ocurrencia (Barceló 2003). Entre los contaminantes emergentes presentes en el ambiente destacan los fármacos, compuestos perfluorados, hormonas, drogas de abuso, y productos de cuidado y de higiene personal. Dentro de los contaminantes emergentes se encuentran también los compuestos llamados disruptores endocrinos (WHO 2012) que han tenido efectos en el ambiente mucho más evidentes como ha sido el efecto imposex en organismos (Hill y Janz 2003). Por lo anterior, se da comienzo a la búsqueda de las localidades con mayores concentraciones de estos contaminantes emergentes, su interacción con las matrices ambientales, así como su biodisponibilidad por su posible efecto sobre los organismos.
Existe una continua introducción de contaminantes emergentes al ambiente debido a su presencia en un sinfín de productos de uso cotidiano en la sociedad, como por ejemplo: productos de cuidado personal (PCP), plaguicidas, hidrocarburos aromáticos policíclicos (HAP), plásticos (bisfenoles y ftalatos), tensoactivos, aditivos industriales, compuestos estrogénicos, entre otros.

Los efectos adversos de los CE presentes en los ambientes acuáticos dependen de la frecuencia y la cantidad de su dosificación, si se encuentran en su forma nativa o como metabolitos y de la matriz ambiental en la que se encuentren (Fent et al. 2006).

En México no hay legislación para los contaminantes emergentes, a diferencia de lugares como Estados Unidos y la Unión Europea (Trasande et al. 2016). Sin embargo, en México se han llevado a cabo estudios de estos compuestos con el fin de evaluar su alcance. Por ejemplo los estudios en agua residual y en suelos del Valle del Mezquital, regados con aguas negras (Pérez et al. 2000, Gibson et al. 2007). Asimismo, se han realizado estudios metodológicos para su identificación y cuantificación en agua residual procedente del tratamiento secundario y sedimentos de la zona chinampera de Xochimilco. (Peña-Álvarez y Castillo-Alanís 2015). Sin embargo, estos esfuerzos no son suficientes para la magnitud del problema que representa la presencia de $\mathrm{CE}$ en el ambiente. Es necesario continuar con estos estudios a fin de evidenciar, como en otros países, su omnipresencia 
e impacto sobre la biota y la salud humana. Además, las dificultades analíticas en el estudio de contaminantes emergentes, hacen que trabajos en el análisis químico de éstos en distintas matrices ambientales, sea de gran trascendencia.

Dentro de las matrices ambientales receptoras de los CE se encuentran los sedimentos. El papel que juegan los sedimentos en el medio acuático es importante, ya que es reflejo de lo que sucede en la columna de agua (Hopkinson et al. 1999). La superficie de las partículas orgánicas y minerales de los sedimentos presenta una alta actividad electrostática y de fuerzas moleculares, lo que implica la existencia de fenómenos como la adsorción de cationes y moléculas orgánicas exógenas. Los sedimentos brindan servicios al ecosistema como almacén o sumidero de carbono, nutrientes y amortiguamiento de contaminantes. Sin embargo, al funcionar como almacén también pueden liberar dichos contaminantes a corto o largo plazo bajo condiciones físicas y químicas cambiantes, resultando en su removilización (Zhu et al. 2014).

Los sedimentos están constituidos por partículas orgánicas e inorgánicas y agua intersticial, comúnmente llamada agua de poro. El agua intersticial es la que ocupa el espacio entre las partículas del sedimento. Está en contacto con éste por largos períodos de tiempo y ocupa más del $80 \%$ del volumen en un depósito de sedimento. Varias de las características y propiedades del agua de poro pueden mostrar el grado de contaminación de un área debido a sus propiedades físicas y químicas, como tiempo de residencia, flujo de partículas, cantidad de materia orgánica, etc. El agua intersticial obtenida de los sedimentos constituye un sumidero de contaminantes y se ha demostrado su utilidad como indicadora de impacto ambiental (Strauch et al. 2008) por su propiedad para acumular metales y compuestos orgánicos. De la misma manera, el agua intersticial puede ser fuente de contaminación al liberar dichos compuestos a corto plazo bajo condiciones físicas y químicas cambiantes de $\mathrm{pH}$, potencial redox, temperatura y actividad microbiana entre otras. Asimismo, el análisis de contaminantes en agua intersticial es de gran importancia debido a que los compuestos que se encuentran en esta martiz se consideran biodisponibles. La biodisponibilidad es un concepto muy importante para determinar la verdadera exposición de los organismos a los contaminantes (Bräunig et al. 2016).

El presente trabajo muestra la optimización y desarrollo de una técnica analítica para el análisis de cuatro contaminantes emergentes en una matriz de gran importancia como es el agua de intersticial de sedimentos. En particular, los sedimentos de la zona de Xochimilco, México es una matriz ambiental compleja por su alto contenido de materia orgánica y por la gran cantidad de compuestos presentes.

\section{MATERIALES Y MÉTODOS}

En este estudio se seleccionaron cuatro representantes de las familias de contaminantes emergentes: un compuesto estrogénico el b-estradiol ( $\geq 98 \%$ Sigma-Aldrich, MO, EUA), que es un compuesto continuamente estudiado, ya que es uno de los disruptores endocrinos de más preocupación por su impacto biológico. Un compuesto antibacteriano, el triclosán (Sigma-Aldrich, MO, EUA) porque su presencia en el ambiente favorece la resistencia bacteriana a los antibióticos en los seres humanos. Un fármaco ácido (FA), el ibuprofeno ( $\geq 98 \%$ Sigma-Aldrich, MO, EUA) que es un producto de venta libre y su dosis terapéutica es relativamente alta. Por último una fragancia, el almizcle cetona (Sigma-Aldrich, MO, EUA) que es utilizado en gran parte de los productos de limpieza y uso de cuidado personal.

\section{Obtención del agua intersticial}

Para la obtención del agua de intersticial, se presenta un debate entre dos tipos de métodos de preparación que pueden ser utilizados: in-situ y ex-situ, ambos métodos cuentan con pros y contras particulares.

El método de extracción in-situ usa "muestreadors de diálisis en equilibrio" o "muestreadores pasivos", que son pequeños envases con una membrana por donde se filtra el agua al estar dentro del sedimento, también utilizan succión por vacío mediante jeringas. El método in-situ tiene una menor interacción entre la muestra y el ambiente manteniendo así la integridad química de las muestras; si bien presenta el inconveniente de ser laborioso y de difícil aplicación (Jonker et al. 2018).

Con el método ex-situ, que seleccionado para este estudio, se obtienen volúmenes de agua intersticial de muestra mayores y a mayor profundidad en la columna de los sedimentos. Asimismo, tiene la ventaja de separar el agua intersticial por métodos sencillos como por ejemplo por centrifugación o filtración con ayuda de algún sistema de succión. Con el método ex-situ se obtienen mayores volúmenes de agua intersticial con un menor esfuerzo y resultados satisfactorios (Winger y Lasier 1991, Sarda y Burton 1995). 


\section{Extracción y análisis de los CE}

La extracción en fase sólida (SPE, por sus siglas en inglés) se realizó con cartuchos Oasis $\mathrm{HLB}^{\circledR} 6 \mathrm{cc}$ $(200 \mathrm{mg}$ x $6 \mathrm{~mL}$ ) en un sistema colector de 12 posiciones. El acondicionamiento de la fase sólida de los cartuchos se ejecutó con $10 \mathrm{~mL}$ acetona grado HPLC seguido de $5 \mathrm{~mL}$ de agua Milli-Q (acidificada con $250 \mu \mathrm{L}$ de ácido acético concentrado). La extracción de los $\mathrm{CE}$ se consiguió al pasar por el cartucho $1000 \mathrm{~mL}$ de muestra acidificada previamente a $\mathrm{pH} 2$ adicionando $\mathrm{H}_{2} \mathrm{SO}_{4}$ concentrado (lo anterior asegura que los compuestos ácidos estén protonados para una eficiente adsorción) y posteriormente secando los cartuchos con aire por $15 \mathrm{~min}$.

La elución del fármaco ácido (ibuprofeno) se obtuvo con $5.5 \mathrm{~mL}$ de solución amortiguadora de bicarbonato de sodio $(\mathrm{pH} 10)$ y acetona $(60: 40 \mathrm{v} / \mathrm{v})$. Posteriormente, la fracción de acetona se evaporó hasta sequedad con un flujo de $\mathrm{N}_{2}$ durante 15 min. Se adicionó aproximadamente ocho miligramos de $\mathrm{Na}_{2} \mathrm{SO}_{4}$ anhidro y se lavó con $2 \mathrm{~mL}$ acetato de etilo. Para separar el $\mathrm{Na}_{2} \mathrm{SO}_{4}$ anhidro, la fase orgánica se transfirió con micropipeta a viales de $2 \mathrm{~mL}$ con septo de doble capa silicona-teflón. Se evaporó con flujo de $\mathrm{N}_{2}$, y se redisolvió con $250 \mu \mathrm{L}$ de acetato de etilo y se guardó en refrigeración para su posterior derivación. Un vez eluído el ibuprofeno, los cartuchos se reacondicionaron con $5 \mathrm{~mL}$ de agua Milli-Q y se secaron con aire durante $1 \mathrm{~h}$ antes de eluir los otros $\mathrm{CE}$ con otro disolvente.

La elución de los CE restantes (almizcle cetona, triclosán, y $\beta$-estradiol) se obtuvo con acetona $2 \mathrm{x}$ $5 \mathrm{~mL}$. Posteriormente se evaporó con aproximadamente $200 \mu \mathrm{L}$ con flujo de $\mathrm{N}_{2}$. Se adicionó $\mathrm{Na}_{2} \mathrm{SO}_{4}$ anhidro y se lavó con $2 \mathrm{~mL}$ de acetato de etilo. Para separar el $\mathrm{Na}_{2} \mathrm{SO}_{4}$ anhidro se transfirió una vez más la fase orgánica a viales de $2 \mathrm{~mL}$ con septo de doble capa silicona-teflón. Nuevamente se evaporó con flujo de $\mathrm{N}_{2}$, y redisolvió con $250 \mu \mathrm{L}$ de acetato de etilo y se guardó para su posterior derivación.

Para la derivación de las fracciones se utilizó la mezcla O-bis-(trimetilsilil) trifluoroacetamida + trimetilsilimidazona 99:1 (BSTFA + TMCS 99:1 Supelco, PA, EUA). Está mezcla derivadora ha sido utilizada por Durán (2009) para el análisis de grupos farmacéuticos ácidos, carbamacepinas y potenciales $\mathrm{CE}$ en agua, asimismo ha sido utilizada en el método analítico llevado a cabo por Gibson y colaboradores (2007).

La preparación de los estándares de $\beta$-estradiol, triclosán, ibuprofeno y almizcle cetona se hizo a partir de las soluciones patrón de cada compuesto a una concentración de $100 \mathrm{mg} / \mathrm{L}$ en acetato de etilo grado HPLC, donde posteriormente se realizó una mezcla de los cuatro a una concentración de $1 \mathrm{mg} / \mathrm{L}$ en $50 \mathrm{~mL}$. Una alícuota de $25 \mu \mathrm{L}$ de la mezcla se derivó agregando $30 \mu \mathrm{L}$ de BSTFA:TMCS 99:1 (v/v), llevando la reacción con calentamiento a $70{ }^{\circ} \mathrm{C}$ en incubadora por un tiempo de 30 min dejando enfriar a temperatura ambiente. Después se adicionó $100 \mu \mathrm{L}$ de acetato de etilo. Esta mezcla y sus posteriores diluciones fueron utilizadas para las curvas, validación del método y estándares adicionados.

Cabe mencionar que uno de los compuestos a analizar no era candidato a la derivación por su estructura (almizcle cetona) pues no cuenta con el sitio de acción para que se lleve la reacción (sililación). Aun así se decidió realizar el método de derivación para todos los compuestos juntos y de esta manera evitar separar el compuesto de la mezcla. El almizcle cetona se identifica en los espectros de masa como el mismo compuesto sin modificaciones por el derivado.

\section{Cuantifiación de los CE}

La cuantificación se realizó en un cromatógrafo de gases acoplado con espectrofotometría de masas (CG-EM) (Hewlett Packard HP G1800C GCD Series II), con una columna capilar HP5-MS marca Agilent de $30 \mathrm{~m} \mathrm{X} 0.25 \mathrm{~mm} \mathrm{X} 0.25 \mu \mathrm{m}$ usando helio como fase móvil con un flujo de $1.0 \mathrm{~mL} / \mathrm{min}$. La inyección de las muestras fue de $1 \mu \mathrm{L}$ de forma manual con ayuda de jeringas cromatográficas Hamilton. La temperatura de inyector fue de $250^{\circ} \mathrm{C}$, la temperatura del detector $300{ }^{\circ} \mathrm{C} \mathrm{y} \mathrm{la} \mathrm{rampa} \mathrm{de} \mathrm{temperatura} \mathrm{fue} \mathrm{desde}$ una temperatura inicial de $100{ }^{\circ} \mathrm{C}$ durante 1 min y a continuación una rampa de $20^{\circ} \mathrm{C} / \mathrm{min}$ hasta $280^{\circ} \mathrm{C}$ y durante de $10 \mathrm{~min}$

Con la mezcla derivada se realizó una curva de calibración de $0.5,5,10,15,20,25,30,35$ y $40 \mu \mathrm{g} / \mathrm{L}$ en acetato de etilo dando una regresión lineal $(\mathrm{R})$ de: ibuprofeno 0.994, almizcle cetona 0.993, triclosán 0.992 y $\beta$-estradiol 0.992 .

\section{Análisis de muestras ambientales}

La zona de estudio se encuentra en el área protegida de la zona chinampera de Xochimilco en el suroeste de la Ciudad de México. Cabe resaltar la importancia de la zona por ser Patrimonio de la Humanidad, por su actividad agrícola, por ser estación de aves migratorios y por sus servicios ambientales a la zona metropolitana. Esta zona es ideal para el estudio de CE, debido a que el agua de los canales, antes lacustre, desde mediados del siglo XX se sustenta principalmente de aguas residuales procedentes del tratamiento secundario. Aunado a lo anterior, está 
el hecho de que el flujo lento del agua (acuitardo) hace que los contaminantes tiendan a acumularse en los sedimentos.

Los canales seleccionados en la zona chinampera de Xochimilco fueron: La Draga (UTM X = 489012; Y = 2130908), Ampampilco (UTM X = 490376; $Y=2131015)$, Tlicuilli (UTM X = 491606; $\mathrm{Y}=2131969)$, Apatlaco (UTM X $=490804 ; \mathrm{Y}$ $=2129991$ ), Texhuilo (UTM X = 490774; $\mathrm{Y}=$ 2131874 y La Asunción (UTM X = 489350; Y = 2130647). El muestreo se realizó en la época seca debido a que supone una mayor acumulación de contaminantes en el fondo de los canales. En los sitios de muestreo se tomaron in-situ los parámetros físicos y químicos del agua (multiparámetro Hanna HI9828, Hanna Instruments, Woonsocket, RI, USA).

La toma de muestras de sedimento fue con una draga tipo Ponar Petitte que obtiene sedimento de hasta $20 \mathrm{~cm}$ de profundidad. Del centro del bocado se tomaron aproximadamente dos litros de sedimento en frascos de vidrio ámbar conservados a $4{ }^{\circ} \mathrm{C}$, por no más de $24 \mathrm{~h}$. En una fracción del sedimento se determinó, en el laboratorio, la concentración de materia orgánica (MO) con un sistema TOC (Apollo 9000, Tekmar-Dohrmann, EUA). Asimismo, se separó el agua intersticial por centrifugación (centrífuga Universal 32, Hettich, Tuttlingen Germany) y filtración con filtros de $1.2 \mu \mathrm{m}$ de tamaño de poro (Whatman de fibra de vidrio grado 934-AH, Buckinghamshire, Reino Unido). De este proceso se obtuvo aproximadamente $1.5 \mathrm{~L}$ del agua intersticial y se almacenó en botellas de vidrio ámbar previamente lavadas con diversos reactivos.

\section{RESULTADOS Y DISCUSIÓN}

Ensayos de elución de la extracción en fase solida

En la literatura son varios los disolventes o medioS de elución que se señalan para usarse con cartuchos SPE, como metanol, acetato de etilo y acetona (Vanderford y Snyder 2006). Gibson y colaboradores (2007) realizaron pruebas con diferentes disolventes, entre ellos el metanol. En su estudio reportan una recuperación aproximada del $90 \%$ en varios analitos.

En este estudio se hicieron ensayos con metanol como disolvente para la elución, pero no se consiguió eluir ninguno de los compuestos de estudio, lo cual se adjudicó a la falta de afinidad del disolvente por los analitos. Partiendo de esto, se decidió utilizar un disolvente menos polar, el acetato de etilo, utilizado en la literatura para la elución de estos compuestos (Gibson et al. 2007). La utilización de este solvente dio resultados satisfactorios, aunque a concentraciones menores a $30 \mu \mathrm{g} / \mathrm{L}$ del $\beta$-estradiol no fue posible cuantificar fácilmente el compuesto debido a variaciones y picos no definidos.

\section{Ensayos de las condiciones cromatográficas}

Se inyectaron los compuestos derivados, a excepción del almizcle cetona, a concentraciones de $1 \mathrm{mg} / \mathrm{L}$ en acetato de etilo en modo de escaneo y se identificaron con ayuda de la base de datos de los espectros de masas (Fig. 1a-e). Esta concentración tan alta sólo se utiliza en el modo escaneo para obtener buenos espectros de masas y que el modo de monitoreo de un sólo ion (SIM, por sus siglas en inglés) sea exitoso.

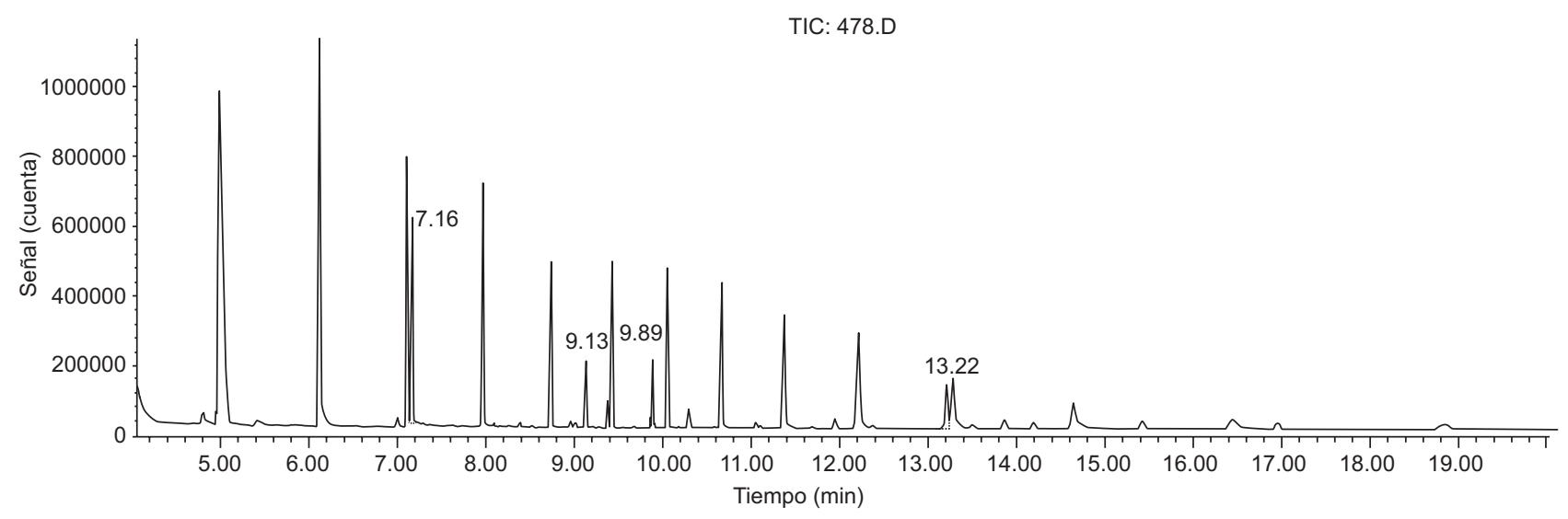

Fig. 1a. Cromatograma en modo escaneo: ibuprofeno (7.1), almizcle cetona (9.1), triclosán (9.8) y $\beta$-estradiol (13.1) 


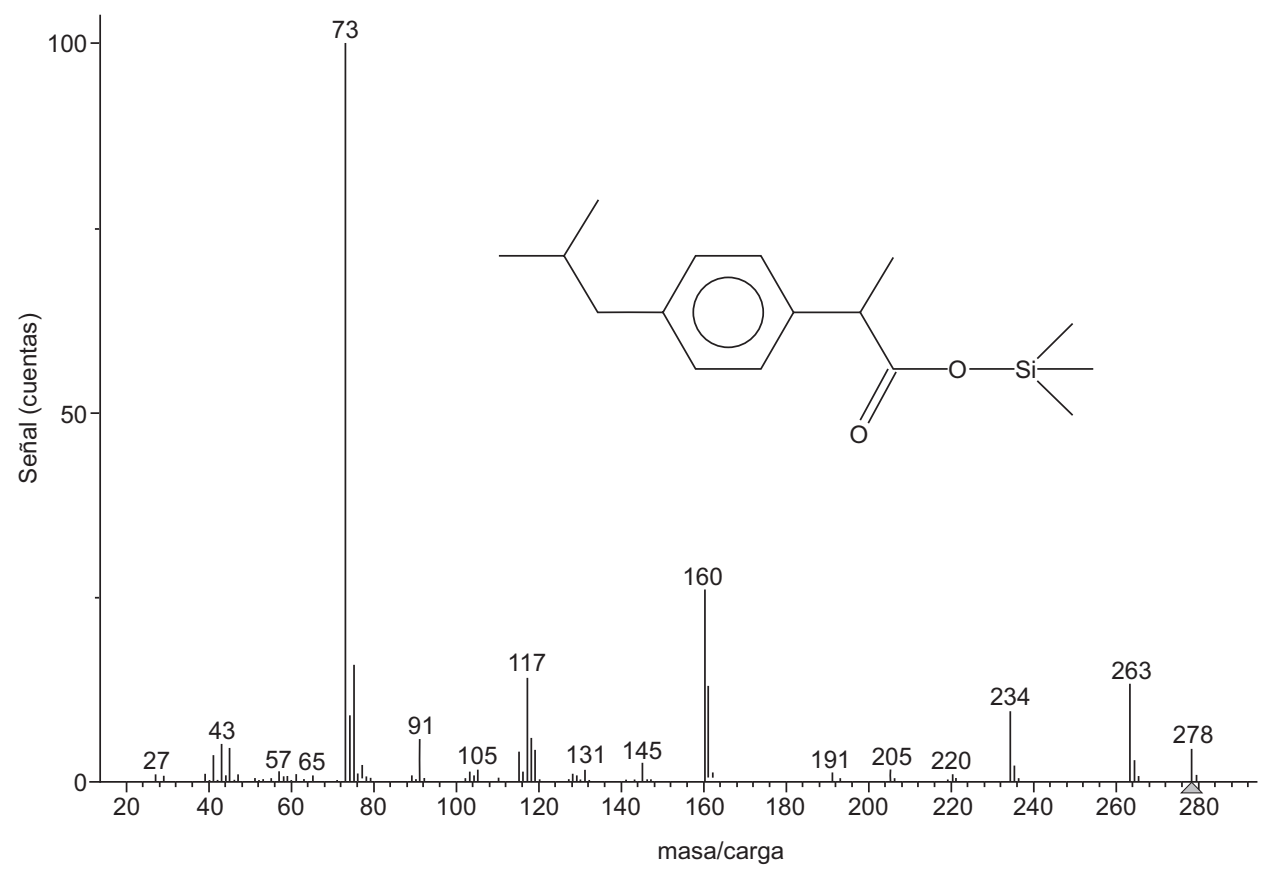

Fig. 1b. Espectro de masas del ibuprofeno derivado

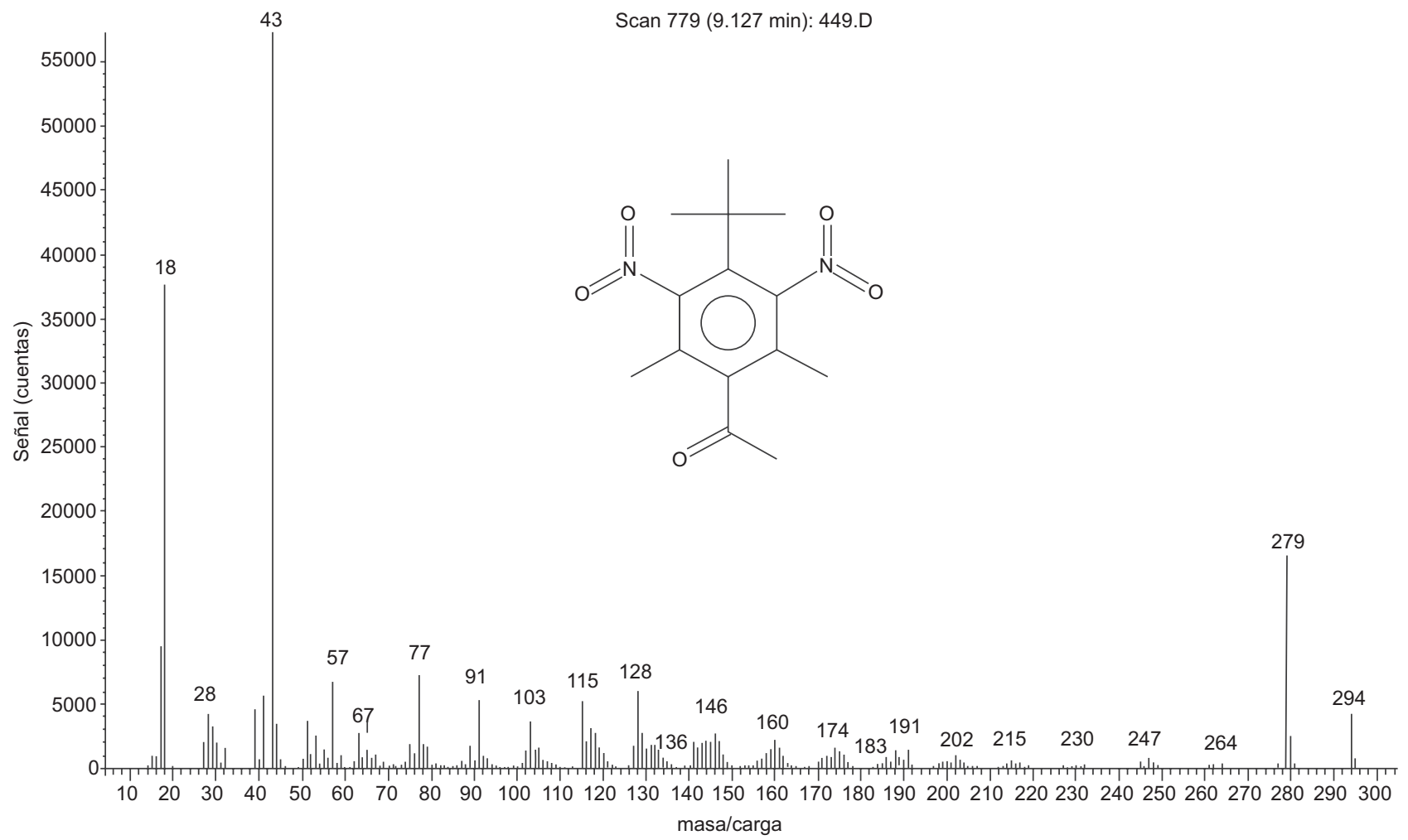

Fig. 1c. Espectro de masas del almizcle cetona (no se puede derivar) 


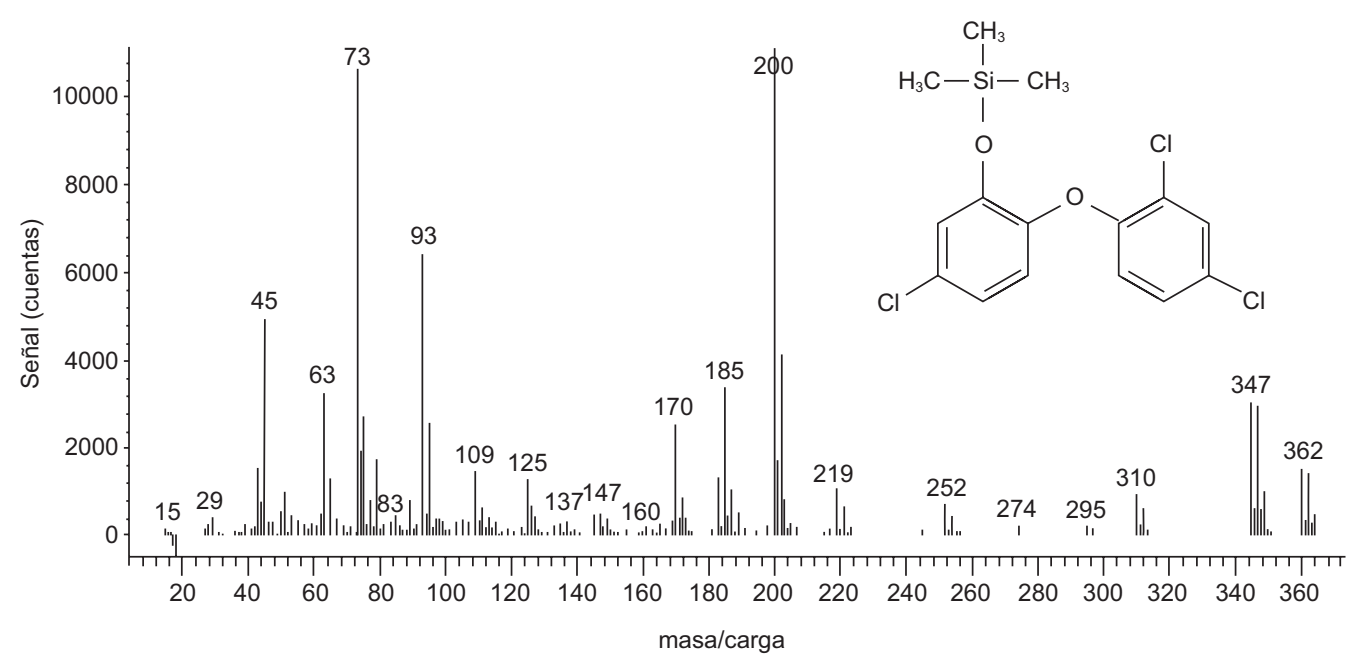

Fig. 1d. Espectro de masas del triclosán derivado

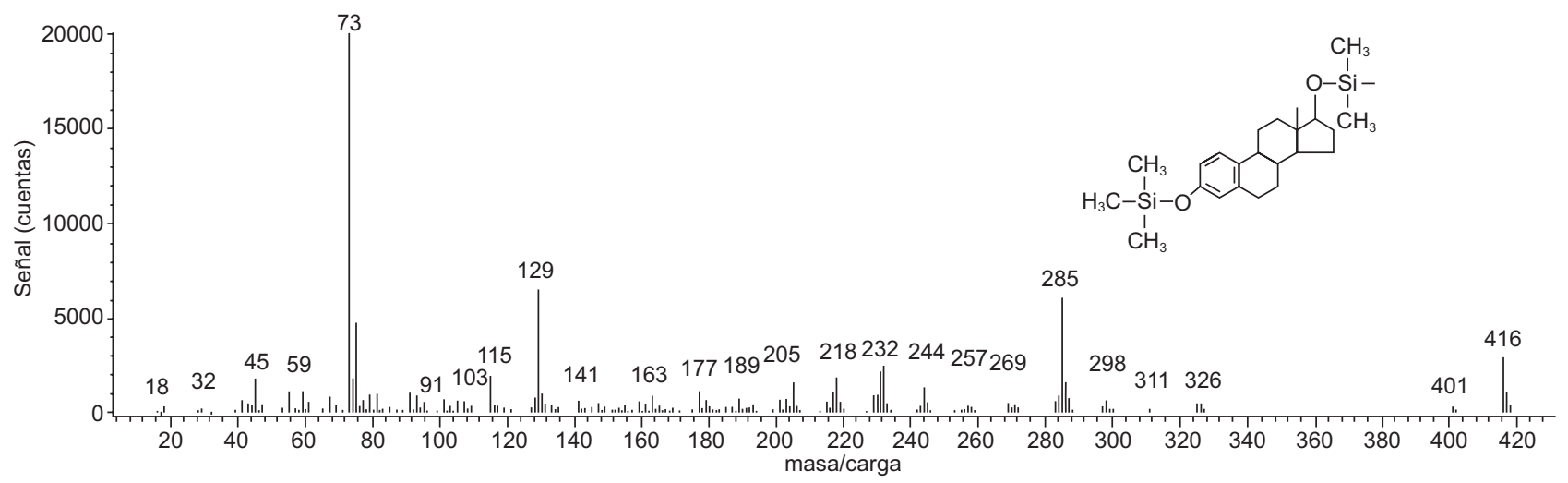

Fig. 1e. Espectro de masas del $\beta$-estradiol derivado

Cabe destacar que durante el análisis se presentaron picos extras en los cromatogramas. Estos picos los podemos definir como residuos que llegaron a reaccionar con el derivado, puesto que la derivación no es una reacción específica y puede interaccionar con residuos dentro del material de laboratorio (ftalatos por ejemplo, en materiales de plástico). Este problema se agrava en muestras ambientales por lo que realizar la detección y cuantificación de los CE en modo escaneo en el CG-EM no es recomendable. Los cromatogramas saturados de picos se pueden simplificar al realizar el análisis cromatográfico en el modo "monitoreo de un sólo ion" (SIM, por sus siglas en inglés). El modo SIM en el sistema CG-EM evita la posible interferencia de otros compuestos y por tanto aumenta la sensibilidad y selectividad del analito. El modo SIM consiste en la búsqueda selectiva por el programa del CG-EM de las masas características de los compuestos que estén presentes en la muestra. Es el modo a emplear para el análisis cuantitativo de trazas de compuestos conocidos como se muestra en el cuadro I y figura 2.

\section{Validación del método}

La validación se logra con el uso de "Proceso de Validación del Método de Laboratorio Único" (Thompson et al. 2002) a través de la determinación de los parámetros de desempeño del método. Estos parámetros incluyen la precisión, la exactitud, linealidad (intervalo de trabajo), el límite de detección, la selectividad y especificidad del método y los estudios de efecto de matriz con muestras adicionadas.

\section{Precisión}

Para determinar la precisión del método, se realizaron cinco repeticiones de agua Milli-Q adicionada con los analitos estudiados a dos concentraciones: 10 y $25 \mu \mathrm{g} / \mathrm{L}$. Las repeticiones del método arrojaron desviaciones estándar relativas de 0.02 y $0.05 ; 0.21$ 
CUADRO I. IONES SELECCIONADOS Y TIEMPOS DE RETENCIÓN DE LOS ANALITOS DE INTERÉS

\begin{tabular}{lcl}
\hline Compuesto & Tiempo de retención & Ion selectivo masas \\
\hline Ibuprofeno & 7.11 & $\underline{\mathbf{2 7 8}}, 263,160$ \\
Almizcle cetona & 9.07 & $\underline{\mathbf{2 9 4}}, 279$ \\
Triclosán & 9.80 & $\underline{\mathbf{3 6 2}}, 200,347$ \\
$\beta$-estradiol & 13.10 & $\underline{416}, 285,401,129$ \\
\hline
\end{tabular}

Masas subrayadas corresponden al ion molecular del compuesto derivado en su caso

y $0.35 ; 0.03$ y $0.07 ; 0.06$ y 0.05 para el ibuprofeno, almizcle cetona, triclosán y $\beta$-estradiol respectivamente. Para comprobar que la precisión de las muestras es adecuada, se calculó el índice HorRat (Horwitz y Albert 2006) que es un parámetro normalizado utilizado como un criterio para indicar la aceptabilidad en la precisión (reproducibilidad) de un método de análisis. De esta manera, se considera que la repetibilidad es aceptable si el índice de HorRat tiene un valor entre 0.5-2. Así, el índice de HorRat se define como:

HorRat $_{\mathrm{r}}=$ DSR (práctica) / DSR (calculada).

Dónde:

DSR $($ práctica $)=$ es la desviación estándar relativa de las repeticiones experimentales

DRS $($ calculada $)=2 \mathrm{C}^{-0.15}$, donde $\mathrm{C}$ es la concentración en $\%$

De esta manera, el índice de HorRat calculado para una concentración de $10 \mu \mathrm{g} / \mathrm{L}$ para los analitos de estudio: ibuprofeno 1.56, almizcle cetona 1.98, triclosán 1.55 y $\beta$-estradiol 1.73 , todos se encuentran dentro del intervalo que se considera un valor aceptable.

\section{Exactitud}

La exactitud del método se obtiene de la recuperación de los analitos de estudio de un estándar. En este caso, dado que no hay estándares de referencia para los analitos y la matriz ambiental estudiados, los experimentos de exactitud se realizaron con los estándares preparados mencionados anteriormente. Los estudios de recuperación se realizaron con la concentración más baja de $10 \mu \mathrm{g} / \mathrm{L}$ de los analitos. El ibuprofeno, el almizcle cetona y el triclosán presentaron recuperaciones del 90.3 , 91.9 y $83.4 \%$, respectivamente. El $\beta$-estradiol presentó recuperaciones del $66.1 \%$ y mostró algunas dificultades al cuantificarse, como inconsistencia en las repeticiones y picos no definidos a concentraciones menores de $30 \mu \mathrm{g} / \mathrm{L}$. Este comportamiento ha sido descrito por otros autores (Durán 2009) concluyendo que estos valores son admisibles por la normativa europea. Por lo anterior, los estudios de exactitud del $\beta$-estradiol, se hicieron a concentraciones de $30 \mu \mathrm{g} / \mathrm{L}$.

\section{Límite de detección y sensibilidad}

El límite de detección se define como la concentración más baja del analito que se puede distinguir inequívocamente del ruido (cero). Por su parte, la sensibilidad es la capacidad del método para cambiar la intensidad de la señal con respecto a la concentración. Sin embargo, no existe parámetro estadístico en la validación que indique la sensibilidad. De ahí que la sensibilidad del método se integra dentro del límite de detección, de tal manera:

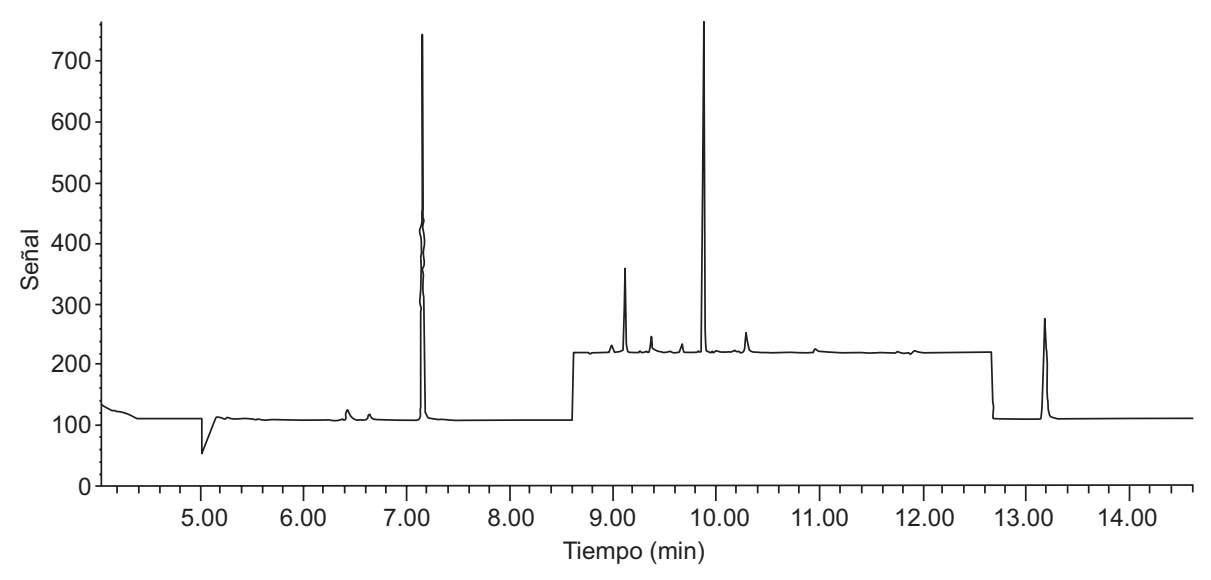

Fig. 2. Cromatograma en modo de monitoreo de un solo ión: ibuprofeno (7.1), almizcle cetona (9.1), triclosán (9.8) y $\beta$-estradiol (13.1) a una concentración de $30 \mu \mathrm{g} / \mathrm{L}$ 
$\mathrm{C}_{\mathrm{LD}}=\mathrm{DS}_{\mathrm{B}} / \mathrm{M}$

Dónde:

$\mathrm{C}=$ concentración o masa límite de detectar.

$\mathrm{m}=$ es la pendiente de la curva de calibración a bajas

concentraciones

DS = Desviación estándar de estándares de agua Milli-Q adicionados

De tal manera, que para determinar el límite de detección se consideró la mínima concentración o masa que se puede detectar utilizando el método que se está validando. Así, los límites de detección en modo SIM para estándares de agua Milli-Q adicionados fueron para el ibuprofeno 0.079; almizcle cetona 0.27 ; triclosán 0.03 y $\beta$-estradiol 0.12 todos en $\mu \mathrm{g} / \mathrm{L}$. Comparando con trabajos similares, Rice y Mitra (2007) encontraron límites de detección del mismo orden de magnitud al de este trabajo. Sus límites de detección fueron de $0.02 \mu \mathrm{g} / \mathrm{L}$ en ibuprofeno y en almizcle cetona $0.26 \mu \mathrm{g} / \mathrm{L}$.

\section{Selectividad y especificidad del método}

La selectividad es la capacidad de un método de distinguir el analito de interés de otras sustancias. En nuestro estudio se aumentó la selectividad de los analitos analizando sólo un compuesto de interés por familia y utilizando un método selectivo como es el monitoreo SIM. En el caso de detecciones cromatográficas, la selectividad se puede expresar como:

$\mathrm{R}_{\mathrm{s}}=\frac{\Delta \mathrm{T}}{0.5\left(\mathrm{~W}_{\mathrm{A}}+\mathrm{W}_{\mathrm{B}}\right)}$

Dónde:

$R_{S}$ es la resolución entre picos cromatográficos $\Delta \mathrm{t}$ es la diferencia de tiempo entre el pico A y el pico B
$\mathrm{W}_{\mathrm{A}}$ es la anchura del pico A en su base

$\mathrm{W}_{\mathrm{B}}$ es la anchura del pico B en su base Quedando la resolución entre picos como: $\Delta \mathrm{t}($ Ibuprofeno - Almizcle cetona $)=1.96$ $\Delta \mathrm{t}($ Almizcle cetona-Triclosán $)=0.73$ $\Delta \mathrm{t}($ Triclosán $-\beta$-estradiol $)=3.3$

Los resultados de la resolución entre picos cromatográficos de este trabajo se presentan en el cuadro II.

CUADRO II. SELECTIVIDAD CROMATOGRÁFICA DEL MÉTODO

\begin{tabular}{lc}
\hline Resolución entre picos & $\Delta \mathrm{t} / 0.5\left(\mathrm{~W}_{\mathrm{A}}+\mathrm{W}_{\mathrm{B}}\right)$ \\
\hline $\mathrm{R}($ Ibuprofeno - almizcle cetona) & 36.98 \\
$\mathrm{R}($ Almizcle cetona-triclosán) & 16.98 \\
$\mathrm{R}($ Triclosan - $\beta$-estradiol) & 57.89 \\
\hline
\end{tabular}

Son tres los factores que influyen en la resolución cromatográfica: la retención, la selectividad y la eficiencia, de tal modo que controlando todas las anteriores se tiene una buena separación de los picos.

\section{Efectos de matriz}

Para verificar los efectos de la matriz que pudiera tener el agua intersticial, se aplicó el método de adición estándar efectuado sobre muestras ambientales de agua intersticial. La adición fue una mezcla de ibuprofeno, almizcle cetona y triclosán a $10 \mu \mathrm{g} / \mathrm{L}$ y $\beta$-estradiol a $30 \mu \mathrm{g} / \mathrm{L}$. El cuadro III muestra las recuperaciones obtenidas para la adiciones en el agua intersticial y agua Milli-Q.

De los resultados de recuperación de las muestras adicionadas (Cuadro III), se puede observar menores porcentajes de recuperación comparado con los

CUADRO III. PORCENTAJE DE RECUPERACIÓN PARA EL AGUA INTERSTICIAL Y MILLI-Q ADICIONADA

\begin{tabular}{|c|c|c|c|c|c|c|c|c|}
\hline & \multicolumn{2}{|c|}{ Ibuprofeno } & \multicolumn{2}{|c|}{ Almizcle cetona } & \multicolumn{2}{|c|}{ Triclosán } & \multicolumn{2}{|c|}{$\beta$-estradiol } \\
\hline & Milli-Q & Intersticial & Milli-Q & Intersticial & Milli-Q & Intersticial & Milli-Q & Intersticial \\
\hline Repetición 1 & 114.6 & 77.4 & 91.4 & 64.8 & 87.1 & 72.3 & 75.8 & 57.2 \\
\hline Repetición 2 & 84.2 & 82.9 & 84.7 & 80.3 & 88.1 & 78.8 & 57.1 & 56.1 \\
\hline Repetición 3 & 78.9 & 75.7 & 89.8 & 83.5 & 92.2 & 81.7 & 70.5 & 52.8 \\
\hline Repetición 4 & 96.6 & 95.1 & 93.9 & 78.1 & 91.4 & 78.1 & 66.6 & 57.9 \\
\hline Repetición 5 & 83.5 & 76.1 & 94.8 & 87.9 & 87.7 & 77.2 & 74.5 & 52.4 \\
\hline Repetición 6 & 83.0 & 80.7 & 91.7 & 69.9 & 84.8 & 80.3 & 72.1 & 50.8 \\
\hline PROM & 90.1 & 81.7 & 91.0 & 77.4 & 88.5 & 78.1 & 69.4 & 54.7 \\
\hline Desv Est & 13.4 & 7.3 & 3.6 & 8.6 & 2.8 & 3.2 & 6.8 & 2.9 \\
\hline
\end{tabular}

PROM= promedio; Desv Est = desviación estándar 
CUADRO IV. PARÁMETROS FÍSICOS Y QUÍMICOS TOMADOS in situ EN LOS CANALES DE LA ZONA CHINAMPERA DE XOCHIMILCO

\begin{tabular}{lcccccccc}
\hline Canal & $\begin{array}{c}\text { Profundidad } \\
(\mathrm{m})\end{array}$ & $\mathrm{pH}$ & $\begin{array}{c}\text { Temp. } \\
{ }^{\circ} \mathrm{C}\end{array}$ & $\begin{array}{c}\mathrm{O}_{2} \\
\mathrm{mg} / \mathrm{L}\end{array}$ & $\begin{array}{c}\text { Salinidad } \\
\mathrm{PSU}\end{array}$ & $\begin{array}{c}\text { Conductividad } \\
\mu \mathrm{S} / \mathrm{cm}\end{array}$ & $\begin{array}{c}\mathrm{SDT} \\
\mathrm{mg} / \mathrm{L}\end{array}$ & $\begin{array}{c}\text { MO sedimento } \\
\%\end{array}$ \\
\hline Apampilco & 1.1 & 8.13 & 21.56 & 2.37 & 0.42 & 857 & 423 & 23.46 \\
Apatlaco & 1.7 & 8.02 & 22.51 & 4.85 & 0.46 & 938 & 470 & 20.66 \\
La Asunción & 1.3 & 7.60 & 20.9 & $\mathrm{ND}$ & 0.43 & 920 & 447 & 27.38 \\
La Draga & 1.2 & 7.58 & 20.87 & 1.32 & 0.43 & 921 & 440 & 18.16 \\
Texhuilo & 1.3 & 8.21 & 22.4 & 6.58 & 0.42 & 854 & 427 & 23.68 \\
Tlicuilli & 0.8 & 7.74 & 21.36 & 3.16 & 0.42 & 856 & 428 & 17.76 \\
\hline
\end{tabular}

$\mathrm{ND}=$ no detectado, $\mathrm{SDT}=$ sólidos disueltos totales $\mathrm{MO}=$ materia orgánica

estándares preparados con agua Milli-Q, indicando que se tienen efectos de matriz aunque no lo suficiente para ser un problema. Las extracciones en el agua intersticial presentan, en general, una mayor variabilidad. Una posible explicación es que la materia orgánica disuelta en el agua intersticial interaccione con los analitos, de tal manera que el disolvente no tenga la suficiente polaridad para la extracción de los analitos ligados a la materia orgánica. Este fenómeno ya ha sido descrito por otros autores (Rice y Mitra 2007, Acosta-Hernández 2010). De cualquier manera, los resultados obtenidos son comparables con las mediciones de ibuprofeno y de almizcle de Rice y Mitra (2007) que obtuvieron un $89 \%$ para el almizcle, triclosán y $\beta$-estradiol y un $25 \%$ para el ibuprofeno.

\section{Análisis de muestras ambientales}

La zona chinampera de Xochimilco está caracterizada por un alto contenido de materia orgánica, con un bajo contenido de oxígeno y una alta capacidad de adsorción de la fase sólida (arcillas, limos y materia orgánica) (Vanegas Pérez et al. 2017). En la zona lacustre de Xochimilco predominan sedimentos arcillosos con arenas de grano fino y alrededor del $50 \%$ de cenizas volcánicas, cuya textura va de arenosa hasta limo-arcillosa y retienen una alta cantidad de humedad (Ficha Informativa de los Humedales de Ramsar FIR 2004).

El cuadro IV muestra las características físicas y químicas del agua de los canales donde se obtuvieron las muestras de sedimento. El canal de mayor profundidad fue Apatlaco con $1.75 \mathrm{~m}$ y Tlicuilli el de menor con $0.8 \mathrm{~m}$. La conductividad se observa relativamente homogénea en todos los canales donde Apatlaco tiene la más alta con $938 \mu \mathrm{s} / \mathrm{cm}$ y Tlicuilli la más baja con 856 $\mu \mathrm{s} / \mathrm{cm}$. El pH va de ligeramente básico a básico, Texhuilo presenta un $\mathrm{pH}$ de 8.21, el más alto, y La Asunción el menor, $\mathrm{pH}$ 7.60, considerados ambos en un intervalo normal. El oxígeno disuelto en Texhuilo, una zona amplia y profunda, fue el más alto, $6.58 \mathrm{mg} / \mathrm{L}$, mientras que La Draga y LaAsunción son los sitios con niveles más bajos de oxígeno $(1.7$ y $0.3 \mathrm{mg} / \mathrm{L}$, respectivamente).

En los sitios descritos se obtuvo el sedimento y se analizó el agua intersticial. Las figuras 3 y 4 muestran ejemplos de los cromatogramas obtenidos de los analitos estudiados utilizando el método validado.

En la cuadro $\mathbf{V}$ se pueden observar los resultados de los compuestos seleccionados para el análisis en el agua intersticial de los sedimentos de Xochimilco. Los análisis muestran que sólo se detectó la presencia de ibuprofeno y el almizcle cetona en todas las muestras. Su presencia se puede explicar por el uso continuo de los productos de cuidado personal (almizcle cetona) (Tavera et al. 2018) y a que el ibuprofeno es el fármaco más utilizado sin prescripción médica contenido en los medicamentos para el dolor con acción antiinflamatoria (ACPM 2011).

Las concentraciones de ibuprofeno en las muestras ambientales oscilan entre los 0.5 y $13.9 \mu \mathrm{g} / \mathrm{L}$, con la mayor concentración en la zona de La Draga. En este sitio se encuentra una descarga de la planta de tratamiento Cerro de la Estrella, lo que puede explicar las altas concentraciones.

El canal de Apatlaco es el sitio con la siguiente concentración más alta de ibuprofeno $(8.2 \mu \mathrm{g} / \mathrm{L})$ y Texhuilo la de menor, aunque se esperaban las mayores concentraciones en los canales de la Asunción y Ampampilco por su alta actividad urbana cercana a los canales.

El almizcle cetona en el sitio La Draga tiene mayor presencia, donde su concentración es de 82.3 $\mu \mathrm{g} / \mathrm{L}$ mientras que en el canal de La Asunción no fue detectado. Este resultado es interesante ya que se esperaba la mayor concentración en este último punto dado que es una sustancia presente en la mayoría de los productos de limpieza y la zona tiene alta actividad urbana. 


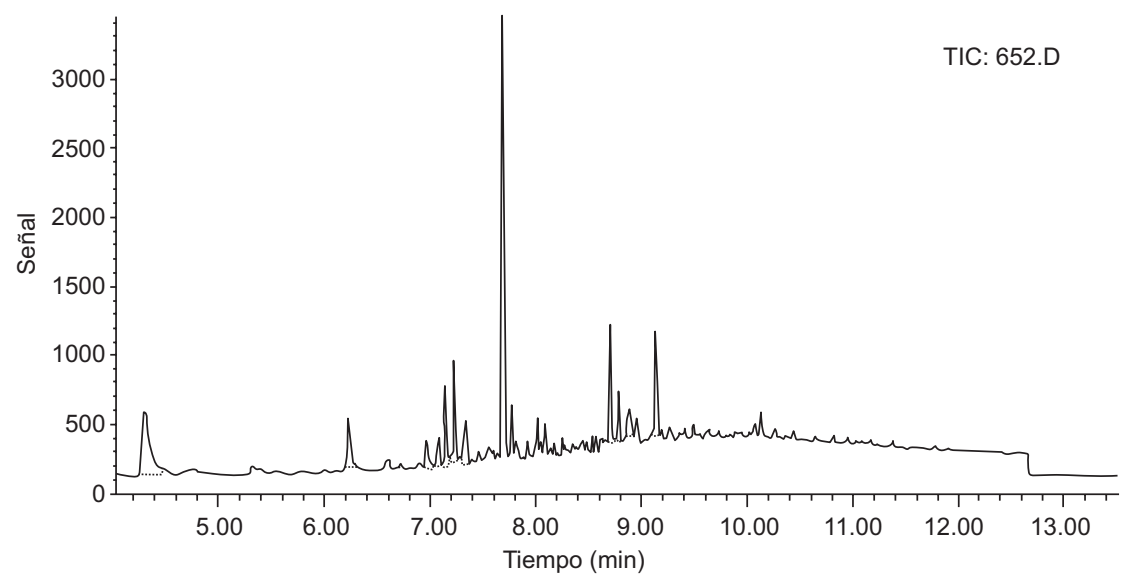

Fig. 3. Cromatograma en modo de monitoreo de un solo ión de la fracción de fármacos ácidos en el agua intersticial de los sedimentos de las muestras ambientales (sitio La Draga)

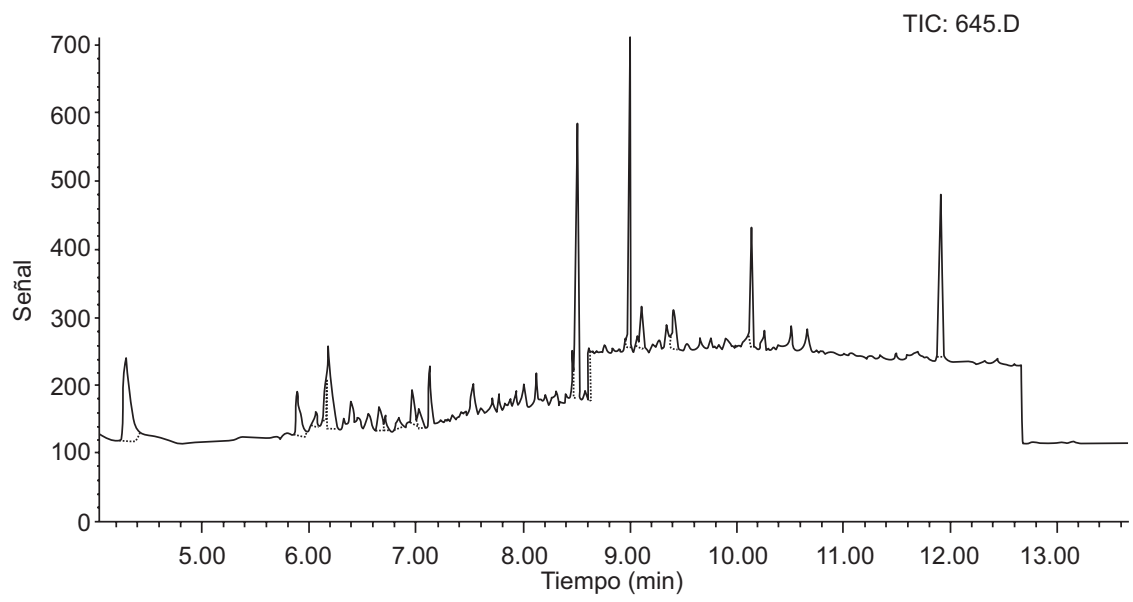

Fig. 4. Cromatograma en modo de monitoreo de un solo ión de la fracción de disruptores endocrinos en el agua intersticial de los sedimentos de las muestras ambientales (sitio La Draga)

CUADRO V. CONCENTRACIONES DE LOS ANALITOS $(\mu \mathrm{g} / \mathrm{L})$ EN EL AGUA INTERSTICIAL

\begin{tabular}{lcccc}
\hline & Ibuprofeno & $\begin{array}{c}\text { Almizcle } \\
\text { cetona }\end{array}$ & Triclosán & $\beta$-estradiol \\
\hline Apatlaco & 8.2 & 57.4 & ND & ND \\
Ampampilco & 0.7 & 11.6 & ND & ND \\
La Asunción & 1.3 & ND & ND & ND \\
La Draga & 13.9 & 82.3 & ND & ND \\
Texhuilo & 0.5 & 4.6 & ND & ND \\
Tlicuilli & 5.2 & 46.9 & ND & ND \\
\hline
\end{tabular}

$\mathrm{ND}=$ no detectado
Al no observarse una relación de las concentraciones de los CE con las características de los sitios, se investigó la relación entre las concentraciones de los $\mathrm{CE}$ detectados y los contenidos de la $\mathrm{MO}$ en los sedimentos. La figura 5 muestra la relación de los CE con la materia orgánica de los sedimentos. La gráfica revela claramente como los $\mathrm{CE}$ y la $\mathrm{MO}$ de los sedimentos muestran una tendencia a correlacionarse de manera inversamente proporcional, especialmente el almizcle cetona. Mu y colaboradores (2013) también observaron una correlación de $\mathrm{r}=0.96$ entre almizcles y MO de sedimentos, y aunque nuestros resultados 

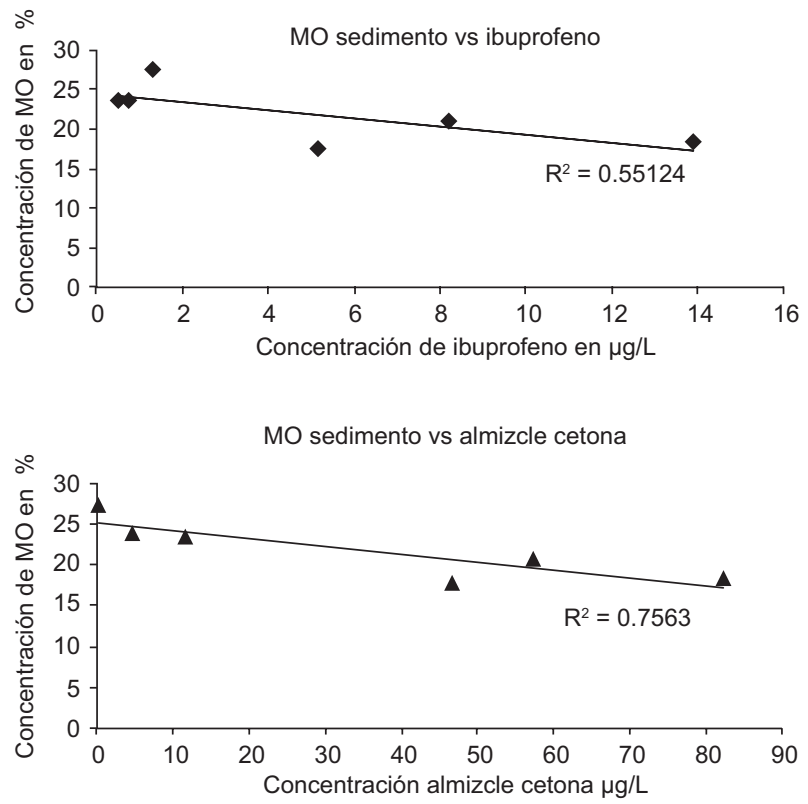

Fig. 5. Relación de los contaminantes emergentes con la materia orgánica (MO) de los sedimentos

no tienen una correlación significativa (por el poco número de repeticiones), sí muestran la tendencia de que el contenido de $\mathrm{MO}$ en sedimentos afecta la disponibilidad de los contaminantes emergentes estudiados.

Estos resultados demuestran la importancia del análisis de contaminantes en el agua intersticial como un indicador de la biodisponibilidad de los contaminantes.

\section{CONCLUSIONES}

El agua intersticial se separó satisfactoriamente a través de la filtración y posterior centrifugación de los sedimentos. El agua intersticial obtenida se hizo pasar a través de cartuchos Oasis HLB ${ }^{\circledR}$ (adsorbente polimérico de fase reversa) donde se obtuvo la retención de los CE. La elución de los analitos tuvo un mejor resultado ejecutándolo en dos pasos: primero con solución amortiguadora de bicarbonato de sodio $(\mathrm{pH} 10)$ y acetona $(60: 40, \mathrm{v} / \mathrm{v})$ para eluir el fármaco ácido (ibuprofeno) y después el resto se eluyó con acetona. Ambas fracciones se combinaron en una sola para realizar la reacción de derivación, aún de aquellos que no se derivan, evitando así contaminación y diferencias. El método implementado tuvo valores de precisión, exactitud, linealidad, selectividad, límites de detección y efectos de matriz admisibles. Con este método se realizaron estudios preliminares de la biodisponibilidad de CE a través de su análisis en agua intersticial de los canales de Xochimilco, México. Con el método fue posible detectar la presencia de ibuprofeno y almizcle cetona y encontrar una relación inversamente proporcional entre los CE y la concentración de $\mathrm{MO}$ en los sedimentos, mostrando así la aplicabilidad del método estudiado.

\section{AGRADECIMIENTOS}

Agradecemos al Instituto EarthWatch, EUA por el apoyo bajo el número de proyecto 8806094 . También agradecemos al M. en C. Antonio Calzada Villafuerte por su valiosa colaboración en la elaboración de las gráficas.

\section{REFERENCIAS}

Acosta-Hernández K.L. (2010). Biodegradación de contaminantes emergentes en columnas empacadas con suelo del valle de Tula. Tesis de Maestría. Universidad Nacional Autónoma de México. Ciudad de México, México, 133 pp.

ACPM (2011). Over-the-counter medications: use in general and special populations, therapeutic errors, misuse, storage and disposal. American College of Preventive Medicine [en línea]. https://c.ymcdn.com/ sites/www.acpm.org/resource/resmgr/timetools-files/ otcmedsclinicalreference.pdf 10/13/2017

Barceló D. (2003). Emerging pollutants in water analysis. Trends Analyt. Chem. 22 (10), Editorial xiv-xvi 14-15. DOI: 10.1016/S0165-9936(03)01106-3

Bräunig J., Tang J.Y.M., Warne M.S.J. y Escherac B.I. (2016). Bioanalytical effect-balance model to determine the bioavailability of organic contaminants in sediments affected by black and natural carbon. Chemosphere 156, 181-190.

DOI: 10.1016/j.chemosphere.2016.04.036

Durán J.C. (2009). Cuantificación de doce contaminanates emergentes provenientes del agua residual empleada para riego en suelos del distrito del riego 03 Tula, Hidalgo. Tesis de Maestría. Universidad Nacional Autónoma de México. Ciudad de México, México, 170 pp.

Fent K., Weston A.A. y Caminada D. (2006). Ecotoxicology of human pharmaceuticals. Aquat. Toxicol. 76 (2), 122-159. DOI: 10.1016/j.aquatox.2005.09.009

Ficha Informativa de los Humedales de Ramsar (FIR) (2004). Zona lacustre "Ejidos de Xochimilco y San Gregorio Atlapulco" [en línea]. http://ramsar.conanp. gob.mx/docs/sitios/FIR_RAMSAR/Distrito_Federal/Xochimilco/Sistema $\% 20$ Lacustre $\% 20$ Ejidos $\% 20$ 
de $\% 20$ Xochimilco\%20y\%20San\%20Gregorio $\% 20$ Atlapulco.pdf 04/03/2016

Gibson R., Becerril-Bravo E., Silva-Castro V. y Jiménez B. (2007). Determination of acidic pharmaceuticals and potential endocrine disrupting compounds in wastewaters and spring waters by selective elution and analysis by gas chromatography-mass spectrometry. $\mathrm{J}$. Chrom. A 1169 (1-2), 31-39.

DOI: $10.1016 /$ j.chroma.2007.08.056

Hill R.L.J. y Janz D.M. (2003). Developmental estrogenic exposure in zebrafish (Danio rerio). Effects on sex ratio and breeding success. Aquat. Toxicol. 63 (4), 417-429. DOI: 10.1016/S0166-445X(02)00207-2

Hopkinson C., Giblin A., Tucker J. y Garritt R. (1999). Benthic metabolism and nutrient cycling along an estuarine salinity gradient. Estuaries 22 (4), 863-881. DOI: $10.2307 / 1353067$

Horwitz W. y Albert R. (2006). The Horwitz ratio (HorRat): A useful index of method performance with respect to precision. J. AOAC Int. 894 (4), 1095-1109.

Jonker M.T.O., van der Heijden S.A., Adelman D., Apell J.N., Burgess R.M., Choi Y., Fernandez L.A., Flavetta G.M., Ghosh U., Gschwend P.M., Hale S.E., Jalalizadeh M., Khairy M., Lampi M.A., Lao W., Lohmann R., Lydy M.J., Maruya K.A., Nutile S.A., Oen A.M.P., Rakowska M.I., Reible D., Rusina T.P., Smedes F. y Yanwen Wu Y. (2018). Advancing the use of passive sampling in risk assessment and management of sediments contaminated with hydrophobic organic chemicals: Results of an international Ex-Situ passive sampling interlaboratory comparison. Environ. Sci. Technol. 52 (6), 3574-3582.

DOI: $10.1021 /$ acs.est.7b05752

Mu L., Li Z. y Wen J. (2013). Distribution characteristics and sorption mechanisms of polycyclic musks in the sediments from Haihe river and Dagu sewage drainage channel. Fresen. Environ. Bull. 22 (9), 2597-2603.

Peña-Álvarez A. y Castillo-Alanís A. (2015). Identificación y cuantificación de contaminantes emergentes en aguas residuales por microextracción en fase sólida-cromatografía de gases-espectrometría de masas (MEFS-CG-EM). Rev. Esp. Cie. Quím. Biol. 18 (1), 29-42. DOI: 10.1016/j.recqb.2015.05.003

Pérez R., Jiménez R., Jiménez B.E. y Chávez A. (2000). ¿El agua del valle del Mezquital, fuente de abastecimiento para el Valle de México?. Memorias. Congreso Nacional de Ingeniería Sanitaría y Ciencias Ambientales. Morelia, Michoacán. 21 al 24 de marzo, 2000. CD-ROM.

Rice S.L. y Mitra S. (2007). Microwave-assisted solvent extraction of solid matrices and subsequent detection of pharmaceuticals and personal care products (PPCPs) using gas chromatography-mass spectrometry. Anal. Chim. Acta 589 (1), 125-132.

DOI: 10.1016/j.aca.2007.02.051

Sarda N. y Burton G.A. (1995). Ammonia variation in sediments: spatial, temporal and methodology related effects. Environ. Toxicol. Chem. 14 (9), 1499-1506. DOI: 10.1002/etc.5620140908

Strauch G., Möder M., Wennrich R., Osenbrück K., Gläser H.R. y Schladitz T. (2008). Indicators for assessing anthropogenic impact on urban surface and groundwater. J. Soils Sediment 8 (1), 23-33.

DOI: $10.1065 /$ jss2007.06.234

Tavera B.I., Tames F., Silva J.A., Ramos S., Homem V., Ratola N. y Carreras H. (2018). Biomonitoring levels and trends of PAHs and synthetic musks associated with land use in urban environments. Sci. Total Environ. 618, 93-100. DOI: 10.1016/j.scitotenv.2017.10.295

Thompson M., Ellison S.R. y Wood R. (2002). Harmonized guidelines for single-laboratory validation of methods of analysis (IUPAC Technical Report). Pure Appl. Chem. 74 (5), 835-855. DOI: 10.1515/ci.2002.24.5.26a

Trasande L., Zoeller R.T., Hass U., Kortenkamp A., Grandjean P., Myers J.P., DiGangi J., Hunt P.M., Rudel R., Sathyanarayana S., Bellanger M., Hauser R., Legler J., Skakkebaek N.E. y Heindel J.J. (2016). Burden of disease and costs of exposure to endocrine disrupting chemicals in the European Union: an updated analysis. Andrology 4 (4), 565-572. DOI: 10.1111/andr.12178

Vanderford B.J. y Snyder S.A. (2006). Analysis of pharmaceuticals in water by isotope dilution liquid chromatography/tandem mass spectrometry. Environ. Sci. Technol. 40 (23), 7312-7320. DOI: 10.1021/es0613198

Vanegas Pérez C., Rosas Pérez I., Cram Heydrich S. y Ponce de León-Hill C.A. (2017). Evaluación de la materia orgánica en la zona lacustre urbano-agrícola de Xochimilco: estrategia para seleccionar puntos focales de descargas de aguas tratadas. Reporte de Proyecto. Secretaría de Ciencia, Tecnología e Innovación, (SECITI). Ciudad de México, México, 124 pp. WHO (2012). State of the science of endocrine disrupting chemicals. Summary for decision-makers. World Health Organization [en línea]. http://apps.who.int/ iris/bitstream/handle/10665/78102/WHO_HSE_PHE_ IHE_2013.1_eng.pdf;jsessionid $=6 \mathrm{C} 6 \mathrm{~EB} 053 \overline{57} \mathrm{AA} \overline{6}$ 5C89A6FBEB7B871546F? sequence $=1$ 08/09/2017

Winger P.V. y Lasier P.J. (1991). A vacuum-operated porewater extractor for estuarine and freshwater sediments. Arch. Environ. Con. Tox. 21 (2), 321-324.

DOI: $10.1007 / \mathrm{BF} 01055353$

Zhu H., Cheng P. y Wang D. (2014). Relative roles of resuspended particles and pore water in release of contaminants from sediment. Water Sci. Eng. 7 (3), 344-350. DOI: 10.3882/j.issn.1674-2370.2014.03.009 